\title{
Suitable energetic conditions for dynamic chemical complexity and the living state
}

\author{
Robert Pascal
}

\begin{abstract}
Any living organism can be considered as a component of a dissipative process coupling an irreversible consumption of energy to the growth, reproduction and evolution of living things. Close interactions between metabolism and reproduction are thus required, which means that metabolism has two main functions. The first one, which is the most easily perceptible, corresponds to the synthesis of the components of living beings that are not found in the environment (anabolism). The second one, which is usually associated with the former, is the dissipative process coupling the consumption of energy to self-organization and reproduction and introducing irreversibility in the process. Considering the origin of life, the formation of at least some of the building blocks constituting a living organism can be envisaged in a close to equilibrium situation under reducing conditions (for instance in hydrothermal vents). However, coupling irreversibly self-organization with the dissipation of an energy flux implies far from equilibrium conditions that are shown in this work to raise quantitative requirements on the height of kinetic barriers protecting metabolites from a spontaneous evolution into deactivated species through a quantitative relationship with the time scale of the progress of the overall process and the absolute temperature. The thermodynamic potential of physical sources of energy capable of feeding the emergence of this capacity can be inferred, which leads to the identification of photochemistry at the wavelength of visible light or processes capable of generating activated species by heating transiently a chemical environment above several thousand Kelvin as the only processes capable of fulfilling this requirement.
\end{abstract}

Keywords: Origin of life, Systems chemistry, Metabolism, Irreversibility, Kinetics

\section{Introduction}

The main features of the living state can be identified even though a comprehensive definition of what is life may be difficult to reach [1] or actually would require an agreement of the scientific community that has not been met yet. It has been proposed that life could be characterized as a kinetic state of matter [2], in which systems that are capable of reproducing themselves can become persistent and evolve owing to the concept of dynamic kinetic stability [3,4]. This description provides a rational basis to the attempt to express natural selection as a physical principle stated almost a century ago by Lotka [5]. The chemical processes capable of initiating life have been recognized decades ago through the analyses of Eigen and coworkers [6,7] as related to the specific

\footnotetext{
Correspondence: rpascal@univ-montp2.fr
}

Institut des Biomolécules Max Mousseron, UMR5247 CNRS - Universités Montpellier 1 \& Montpellier 2, CC17006, Place E. Bataillon, F-34095, Montpellier, France behavior of reaction networks behaving as autocatalysts or more generally hypercycles. These systems correspond in fact to reaction cycles involving multiple feedback processes so that the overall system present unique properties. Although it is possible to understand the emergence of life as that of a genetic replicator [8], and that of the exponential growth needed for these systems to develop $[9,10]$, a metabolic contribution to this process remains unavoidable since autocatalysis does not result in growth for systems close to equilibrium [6], which means that the systems requires the availability of energy-rich building blocks that are used up irreversibly. The metabolic features of living systems have been analyzed by Schrödinger as the need of an association with processes generating an increase in entropy to compensate for the local decrease associated with self-organization [11]. The involvement of processes in which a flux of energy (or matter in an activated state) is irreversibly transformed through a dissipative process, producing entropy in the environment in a 
way that is coupled to a local decrease within the selforganizing system, is then crucial for living organisms. With regard to the origin of life, the formation of an organized system coupling the use of this irreversible energy flux with self-organization must have required the spontaneous decay of chemical species involved in the process to be slow enough so that features of organization can develop. Eschenmoser considered indeed that the complexity of a reaction network involving metabolic cycles or autocatalytic networks associated with the first developments of life must have emerged from systems evolving in a chemical environment held far from equilibrium by kinetic barriers [12-14]. This idea simply means that chemical self-organization cannot emerge when species decay with fast rates toward the equilibrium state. In this essay, this idea is developed in a quantitative way to demonstrate that it can actually lead to valuable conclusions on the thermodynamic potential needed to bring about the living state of matter and on the nature of the corresponding processes. This does not mean that life and evolution can be understood in a deterministic way, but simply that having a probability different from zero for life to emerge requires specific chemical conditions. These requirements must be taken into account when tackling the questions of the origin of living systems on the early Earth or of its possible occurrence on extrasolar planetary systems, but also when considering the evolution of artificial chemical systems based on dynamic kinetic stability [3,4], which constitutes one of the goals of systems chemistry $[15,16]$.

\section{Discussion}

Any species involved in a chemical system involving features of self-organization must be protected from a fast spontaneous decay leading rapidly to deactivated products or side products (Figure 1). This means that every species involved in the proto-metabolic part of Figure 1 must be located in a free energy well and protected by kinetic barriers to avoid its spontaneous fast conversion into close to equilibrium (inactivated) products. This is true for any metabolite that has a lifetime significant compared with the time scale at which the system progresses, a quantity that is related, in the case of metabolic loops, to the turnover frequency proposed as an essential parameter in the kinetic description of catalytic cycles [17]. Transition state theory [18] provides a relationship between the rate constant of the chemical reaction, the free energy of activation (kinetic barrier) and the absolute temperature. The Eyring equation (Equation 1) expresses the rate constant of the transformation of any reactant as a function of the barrier and absolute temperature.

$$
k=\frac{k_{B} T}{h} e^{-\Delta G^{ \pm} / R T}
$$

This relation involves only three variables (the rate constant, the kinetic barrier and the absolute temperature) and universal constants of physics (namely the Boltzmann, Planck and gas constants). It can be transformed to express the height of the kinetic barrier as a function of the half-life of the reaction for a first-order (or pseudo-first order) reaction and of the temperature (Equation 2).

$$
\Delta G^{\ddagger}=R T \ln \frac{k_{B} T t_{1 / 2}}{h \ln (2)}
$$

A linear plot corresponding to equation 2 is displayed in Figure 2 using a logarithmic scale for half-lives covering the full range of chemical times representing ca. 30 orders of magnitude, from the duration of a vibration to

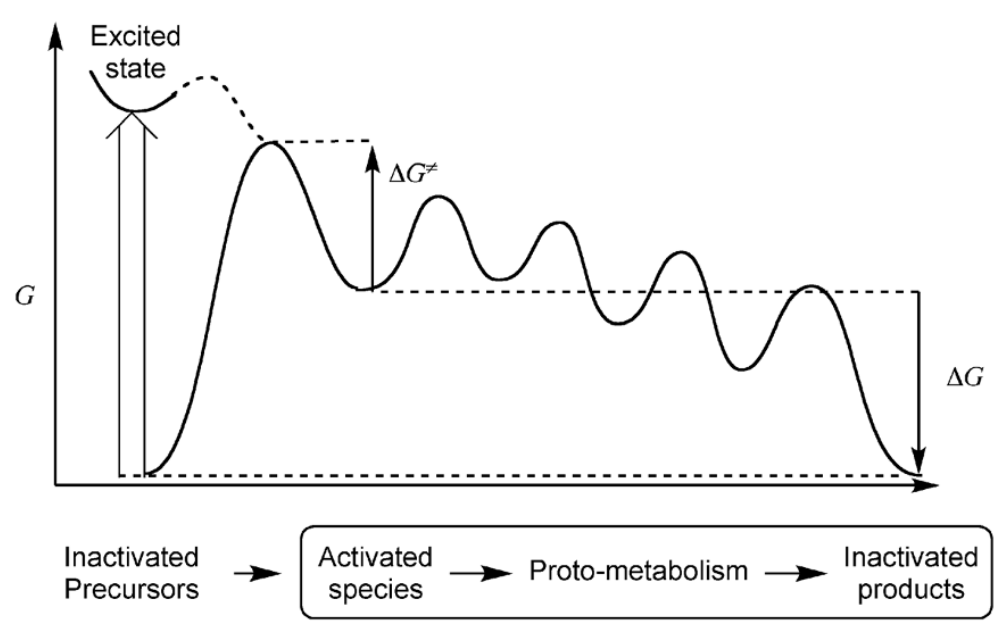

Figure 1 Free energy diagram corresponding to a metabolic pathway in which irreversibility is ensured by the kinetic barrier $\left(\Delta G^{\ddagger}\right)$ of the reverse reaction of the activated species back into inactivated precursors. 

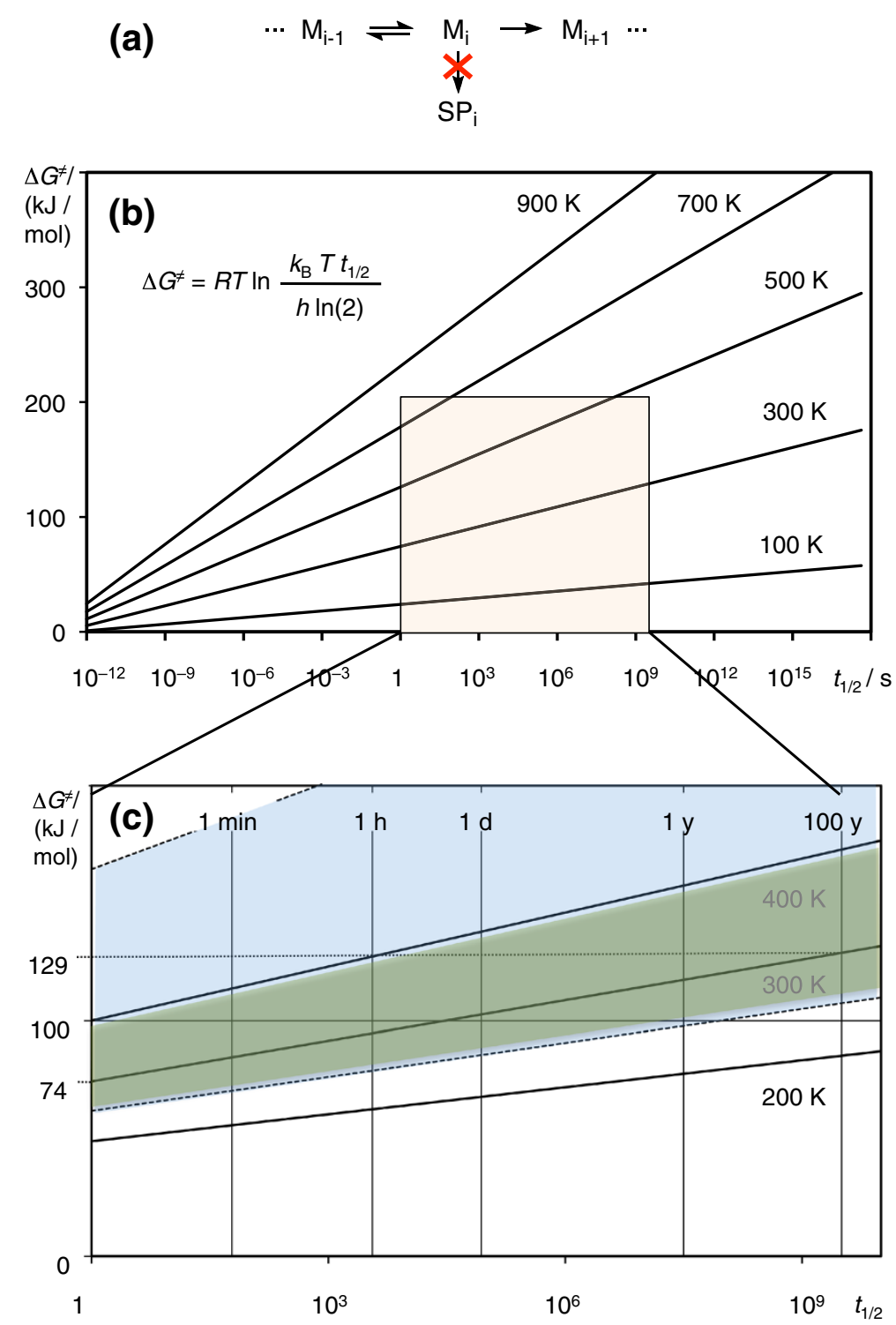

Figure 2 The prediction of the conditions for the emergence of complexity and life from the depth of chemical time. (a) Any kind of metabolite (Mi) involved in a proto-metabolic process must be protected by kinetic barriers to avoid side-reactions (yielding side-products as SPi) or direct reactions to products annihilating features of organization (catalysis, autocatalysis). These barriers help in holding the system far from equilibrium, which is a prerequisite for self-organisation. (b) Transition state theory is used here to predict the heights of these kinetic barriers at given values of the absolute temperature $T$ and for a range of half- lives of the metabolite. Therefore, a requirement for the emergence of complexity and life is established from this relationship connecting the height of the barrier to the lifetime of chemical intermediates for a given absolute temperature. Thirty orders of magnitude separate the lifetime of a transition state (a vibration lasting a fraction of picosecond) from that of the universe (13.7 billion years). A representative range of $1 \mathrm{~s}$ to 100 years (more than nine orders of magnitude or ca. 30\% of the full logarithmic scale) is selected as realistic for chemical intermediates that accumulate in protometabolic processes. (c) Because of the logarithmic dependence, this wide range of lifetimes corresponds to barriers spanning only from 74 to $129 \mathrm{~kJ} \mathrm{~mol}^{-1}$ at moderate temperature (300 Kelvin). The domain of stability of liquid water (from the liquid state at low temperatures and high pressure to the critical point of water, blue area) and that in which forms of extant life on Earth are capable of growth (green area) are also displayed showing that the emergence of life was less unlikely at values of the temperature close to that of the fusion of ice.

the age of the Universe. Considering that the practical lifetime of an energy carrier or a metabolite that accumulates significantly in a metabolic or proto-metabolic pathway must be comprised between $1 \mathrm{~s}$ to $100 \mathrm{yr}$ (more than 9 orders of magnitude) an assessment of the kinetic barriers needed for a system capable of self- organization at moderate temperature $(300 \mathrm{~K})$ can be given as a range of free energy of 74 to $129 \mathrm{~kJ} \mathrm{~mol}^{-1}$ (Figure 2). Because of the logarithmic dependence, this range is astonishingly limited by comparison with the nine-orders in 
magnitude change in lifetimes so that the assessment of this range of kinetic barriers can be considered as representative of the self- organization process through protometabolic pathways. This range of about $100 \mathrm{~kJ} \mathrm{~mol}^{-1}$ can be compared with the bonding energies involved in chemical interactions and amounts to a significant fraction (about one fourth for a C-C bond) of the free energy of a covalent bond. It results that self-organization in systems based on simple molecules must be based on strong chemical bonding. It should not be achievable though weak chemical interactions (Van der Waals forces, hydrogen bonding, hydrophobic interactions...) except for the rare systems based on multivalent bonds and in which bonding energy could be additive [19], because of an adapted rigid structure implying that all bonds are broken in a concerted way. Long-lived species feeding the system in energy and that have to migrate in the environment from the location of their formation to that of the self-organizing chemical system must then be protected from a spontaneous deactivation into products or sideproducts by a free energy barrier $\Delta G^{\neq}$with a similar height (Figure 1). Importantly, this condition also applies to the reverse transformation into their inactivated precursors (Figure 1), which is actually identical to a condition for the process to be irreversible. In other words, the kinetic barrier of the reverse of the activation reaction must be high enough so that the proto-metabolism works as a one-way chemical system. An estimate of the free energy needed for biochemical carriers to enable the development of biochemistry has been made independently to a minimum value of ca. $-50 \mathrm{~kJ} \mathrm{~mol}^{-1}$ (corresponding to $\Delta G$ in Figure 1) [20]. The free energy source capable of driving the system must thus be capable of delivering a thermodynamic potential reaching the sum of the absolute values of $\Delta G^{\neq}$and $\Delta G, c a .150 \mathrm{~kJ} \mathrm{~mol}^{-1}$, which establishes a severe constraint on the thermodynamics of protometabolic systems.

The driver process delivering energy and enabling the formation of an activated carrier in the first step of Figure 1 may be physical (for instance photochemical and not being subject to microscopic reversibility as proposed in an analysis of autocatalytic models on the emergence of homochirality [21]) or chemical. However, its actual nature does not matter. The condition that the proto-metabolisms must work irreversibly (as a one-way flux of reactants) for coupling self-organization with an entropy producing process implies that this driver process is capable of bringing the system into an excited state with a free energy exceeding the level of the transition state of the reaction reverting the energy carrier into its precursors. As a result, this short-lived activated state either reverts back to the reactants or proceeds downhill in the forward direction until a free energy well is reached yielding a chemical carrier conserving a significant part of the free energy of the source. Irreversibility in the proto-metabolic process thus emerges from the kinetic barrier of a reverse reaction. This discussion helps in identifying processes enabling the formation of the energy carriers. Photochemical processes able to deliver the corresponding amount of energy (ca. $150 \mathrm{~kJ}$ $\mathrm{mol}^{-1}$ ) correspond to wavelength of ca. $0.8 \mu \mathrm{m}$ meaning that light in the visible spectrum is needed. Few other physical processes are capable of driving such systems in a spontaneous way. Using the black body emission as a typical example of irreversible transformation of thermal energy, heating a solid to $3600 \mathrm{~K}$ enables the emission of electromagnetic radiations with a maximum at the wavelength of $0.8 \mu \mathrm{m}$. This can be a measurement of the temperature needed for thermal energy to generate activated chemical intermediates, though the system needs a quenching step to preserve the corresponding species from evolving back to equilibrium. The only obvious processes compatible with the effect of heat are lightning, as mimicked in the Miller experiment [22], and impact-shock syntheses [23]. These processes are capable of heating locally and transiently the atmosphere at temperatures exceeding several thousand of $\mathrm{K}$ needed to generate radicals and ions that have enough time to recombine after cooling within a fraction of second at the lower temperature of the high atmosphere. By contrast, finding means by which geothermal energy could directly play a similar role is more problematic.

In principle, having a chemical environment in which species are held far from equilibrium by kinetic barriers may enable certain catalysts to be amplified provided that the reaction network is additionally capable of producing them. These protometabolic cycles involving catalysts that are themselves reproduced by the system are likely to increase the rate of consumption of energy carriers so that they will tend to predominate in the environment-they are selected-, corresponding to the definition of dynamic kinetic stability, which is the main feature of living systems.

\section{Conclusion}

In summary, an analysis starting by considering the fate of any single metabolite shows that the lifetime corresponding to the evolution of the whole chemical system (related to that of its major intermediates) and the absolute temperature determine the heights of the free energy barriers required for protecting metabolites with a significant lifetime from a spontaneous breakdown. At moderate temperatures corresponding to that of the surface of the Earth, these barriers represent a significant part of the dissociation energies of simple covalent bonds signifying that scaffolds based on this kind of linkages are the easiest solution to sustain life under these 
conditions. This analysis establishes that not all forms of energy are capable of sustaining the origin of life and predicts photochemistry or transient heating to temperatures beyond several thousands of Kelvin (by lightning or impacts) as likely sources. Therefore, photochemistry may have been directly involved in the emergence of living organisms or may have been introduced very early in their metabolism long before photosynthesis brought about the possibility of generating both chemical energy under the form of ATP and reducing power used for the biosynthesis of their organics components. As life tend to occupy every ecological niche in which energy is available, further biochemical machineries evolved that use complex mechanisms to extract energy from diluted sources, which may even include syntrophic cooperation of different forms of life that develop different kinds of metabolisms [24]. But, life must have initially be based on much simpler systems so that the requirements defined here introduce new limits for the habitability of the early Earth environments or of extrasolar planets as factors conditioning the origin of life. Chemical principles are thus essential for understanding how life can emerge and introduce independent conditions for habitability that are more strict than those coming from the possibility of the persistence of evolved living beings deduced by analogy to extant life on Earth. The analysis of these principles is also useful in building a theoretical framework for systems chemistry [25].

\section{Competing interests}

The author declares that he has no competing interests.

\section{Acknowledgements}

The author thanks the interdisciplinary program of the CNRS Planetary Environments and Origins of Life (EPOV) for support and the COST Action CM070 "Systems chemistry" for providing a fruitful context for scientific exchanges during the realization of this work.

Received: 2 April 2012 Accepted: 3 May 2012

Published: 17 May 2012

\section{References}

1. Bruylants G, Bartik K, Reisse J: Prebiotic chemistry: a fuzzy field. CR Chimie 2011, 14:388-391.

2. Pross A: Stability in chemistry and biology: Life as a kinetic state of matter. Pure Appl Chem 2005, 77:905-1921.

3. Pross A: Seeking the Chemical Roots of Darwinism: Bridging between Chemistry and Biology. Chem Eur J 2009, 15:8374-8381.

4. Pross A: Toward a general theory of evolution: Extending Darwinian theory to inanimate matter. J Syst Chem 2011, 2:1.

5. Lotka AJ: Natural selection as a physical principle. Proc Natl Acad Sci USA 1922, 8:151-154.

6. Eigen M: Selforganisation of matter and the evolution of biological macromolecules. Naturwissenschaften 1971, 58:465-523.

7. Eigen $M$, Schuster $P$ : The hypercycle. A principle of natural selforganization. Part A. The emergence of the hypercycle. Naturwissenschaften 1977, 64:541-565.

8. Pross A: Causation and the origin of life. Metabolism or replication first? Orig Life Evol Biosph 2004, 34:307-321.

9. Patzke V, von Kiedrowski G: Self replicating systems. Arkivoc 2007, Part 5:293-310.
10. Szathmáry E, Gladkih I: Sub-exponential growth and coexistence of nonenzymatically replicating templates. J Theor Biol 1989, 138:55-58.

11. Schrödinger E: What is life. McMillan: New York; 1946.

12. Eschenmoser A: Chemistry of potentially prebiological natural products. Orig Life Evol Biosph 1994, 24:389-423.

13. Eschenmoser A: Question 1: Commentary Referring to the Statement "The Origin of Life can be Traced Back to the Origin of Kinetic Control" and the Question "Do You Agree with this Statement; and How Would You Envisage the Prebiotic Evolutionary Bridge Between Thermodynamic and Kinetic Control?" Stated in Section 1.1. Orig Life Evol Biosph 2007, 37:309-314.

14. Eschenmoser A: Etiology of potentially primordial biomolecular structures: from vitamin B12 to the nucleic acids and an inquiry into the chemistry of life $\ni$ s origin: a retrospective. Angew Chem Int Ed 2011, 50:12412-12472.

15. Kindermann M, Stahl I, Reimold M, Pankau WM, von Kiedrowski G: Systems chemistry: kinetic and computational analysis of a nearly exponential organic replicator. Angew Chem Int Ed 2005, 44:6750-6755.

16. Ludlow RF, Otto S: (2008) Systems chemistry. Chem Soc Rev 2008, 37:101-108.

17. Kozuch S, Shaik S: How to conceptualize catalytic cycles? The energetic span model. Acc Chem Res 2011, 44:101-110.

18. Kreevoy MM, Truhlar DG: Transition State Theory. In Investigation of rates and mechanisms of reactions, Vol. 6, Part 1. 4th edition. Edited by Bernaconi CF. New York: John Wiley and Sons; 1986:13-95.

19. Jencks WP: On the attribution and additivity of binding energies. Proc Natl Acad Sci USA 1981, 78:4046-4050.

20. Pascal R, Boiteau L: Energy flows, metabolism and translation. Philos Trans R Soc B 2011, 366:2949-2958.

21. Blackmond DG, Matar OK: Re-examination of reversibility in reaction models for the spontaneous emergence of homochirality. J Phys Chem B 2008, 112:5098-5104.

22. Miller SL: A production of amino acids under possible primitive earth conditions. Science 1953, 117:528-529.

23. Chyba C, Sagan C: Endogenous production, exogenous delivery and impact-shock synthesis of organic molecules: an inventory for the origins of life. Nature 1992, 355:125-132.

24. Schink B: Energetics of syntrophic cooperation in methanogenic degradation. Microbiol Mol Biol Rev 1997, 61:262-280.

25. Pascal R: Life metabolism and energy. In Physical Chemistry in Action: Astrochemistry and Astrobiology. Edited by Smith IWM, Cockell CS, Leach S. Dordrecht: Springer; 2012.

doi:10.1186/1759-2208-3-3

Cite this article as: Pascal: Suitable energetic conditions for dynamic chemical complexity and the living state. Journal of Systems Chemistry 2012 3:3.

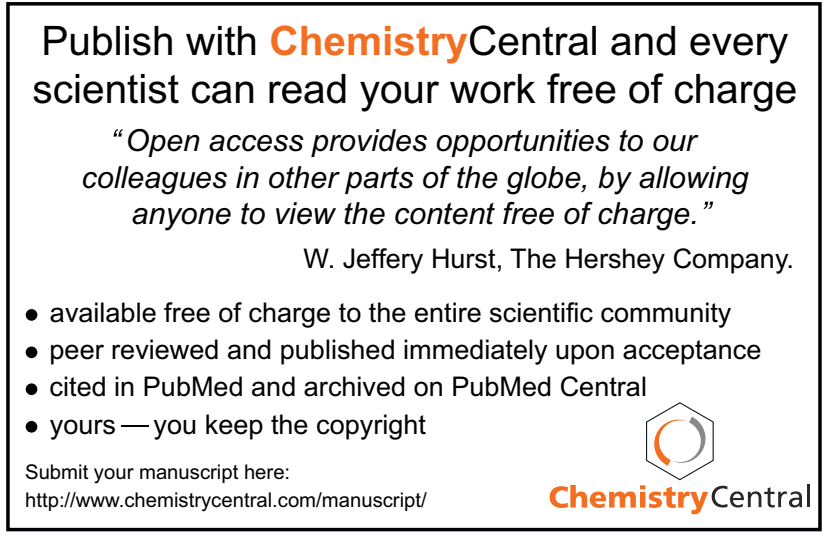

